\title{
Three-particle quantization condition in a finite volume: 1 . The role of the three-particle force
}

\author{
Hans-Werner Hammer, ${ }^{a, b}$ Jin-Yi Pang $^{c}$ and Akaki Rusetsky ${ }^{c}$ \\ ${ }^{a}$ Institut für Kernphysik, Technische Universität Darmstadt, \\ Schloßgartenstraße 2, D-64289 Darmstadt, Germany \\ ${ }^{b}$ ExtreMe Matter Institute EMMI, GSI Helmholtzzentrum für Schwerionenforschung, \\ Planckstraße 1, D-64291 Darmstadt, Germany \\ ${ }^{c}$ Helmholtz-Institut für Strahlen- und Kernphysik (Theorie) \\ and Bethe Center for Theoretical Physics, Universität Bonn, \\ Nußallee 14-16, D-53115 Bonn, Germany \\ E-mail: hammer@theorie.ikp.physik.tu-darmstadt.de, \\ pang@hiskp.uni-bonn.de, rusetsky@hiskp.uni-bonn.de
}

ABSTRACT: Using non-relativistic effective Lagrangians in the particle-dimer picture, we rederive the expression for the energy shift of a loosely bound three-particle bound state of identical bosons in the unitary limit. The effective field theory formalism allows us to explicitly investigate the role of the three-particle force. Moreover, we discuss relaxing the unitary limit of infinite scattering length and demonstrate a smooth transition from the weakly bound three-particle state to a two-particle bound state of a particle and a deeply bound dimer.

KEYWORDS: Lattice field theory simulation

ARXIV EPRINT: 1706.07700 


\section{Contents}

1 Introduction $\quad 1$

2 Two-body bound state 3

3 Dimer formalism in the infinite volume 5

3.1 The Lagrangian 5

$\begin{array}{ll}3.2 & \text { The bound-state equation and the normalization condition }\end{array}$

3.3 Minlos-Faddeev solution 8

3.4 Asymptotic normalization coefficient 8

4 Particle-dimer bound state in a finite volume $\quad 10$

$\begin{array}{ll}\text { 4.1 Expression of the first order finite-volume energy shift } & 10\end{array}$

$\begin{array}{ll}4.2 & \text { Evaluation of the first-order energy shift } \\ \end{array}$

5 Beyond the unitary limit $\quad \mathbf{1 3}$

5.1 A shallow bound state of a particle and a deeply bound dimer 13

$\begin{array}{ll}5.2 \text { A shallow bound state of three particles } & 13\end{array}$

$\begin{array}{lll}5.3 & \text { Subleading contributions } & 14\end{array}$

6 Conclusions 14

$\begin{array}{ll}\text { A Two-body problem using power divergence subtraction } & 15\end{array}$

$\begin{array}{ll}\text { B Calculation of the integrals } & 16\end{array}$

$\begin{array}{ll}\text { C Wave function } & 17\end{array}$

$\begin{array}{ll}\text { C.1 Endpoint singularities } & 17\end{array}$

$\begin{array}{lll}\text { C.2 Pinch singularities } & 19\end{array}$

\section{Introduction}

For some time now lattice QCD calculations have been addressing hadron physics problems which involve the dynamics of three or more hadrons. As an example, we quote the calculation of the parameters of the Roper resonance [1-10], which decays - at a substantial rate - into a nucleon and two pions. The finite-volume effects in such few-body systems are expected to be rather pronounced. Hence, understanding these effects is a necessary precondition for investigating intriguing puzzles such as the level ordering of the $N^{*}(1440)$ and the $N^{*}(1535)$. Another reason for studying the three-body dynamics in a finite volume 
now is the recent advent of lattice QCD calculations of light nuclei [11-13] and corresponding calculations in nuclear effective field theory on the lattice [14-17]. In order to fully exploit these advances, a formalism is needed to translate the "raw" lattice results into physical observables like cross sections into various two- and three-body channels, etc. It is also important that the proposed formalism is not overly complicated and can be used even when only a few data points are available.

The quantization of the energy levels of a three-particle system in a finite volume has been considered first in ref. [18]. In a series of subsequent papers by different groups [19-28] further important aspects of the problem have been addressed. We would like to stress, however, that despite the substantial progress made, the formalism is still very complicated and, in our opinion, not quite ready to be used straightforwardly by lattice practitioners (in contrast to, e.g., the Lüscher equation for two-particle elastic scattering [29]). In addition, the relation between the different approaches is not obvious and has not been discussed in the literature so far. At the same time, in refs. [30-33], the volume dependence of the discrete three-body spectrum has been investigated for bosons as well as nucleons by solving the bound state equations in a finite volume numerically. An effective field theory in the dimer formalism has been used to derive the finite volume bound state equations and relate the bound state properties to scattering parameters in the infinite volume, which greatly simplifies the handling of the three-body problem. These studies also suggest a strategy for formal investigations of three-body dynamics in a finite volume.

The aim of our work is to provide a simple formalism for the analysis of the present and forthcoming lattice data in a straightforward and transparent manner, and to understand the link with the earlier approaches. To this end, we use the particle-dimer approach, which is very convenient and allows one to achieve this goal with a surprising ease. We also found it justified to split the material in two parts. In the first paper, we consider a very simple system - a shallow three-body bound state in a finite volume - which has been already studied in the literature [19, 27]. A leading-order analytic expression for the finite-volume energy shift of this system in the unitary limit is available, and we shall see how this result can easily be obtained in the particle-dimer picture. In addition, we address the following issues which were not considered in previous work:

(i) The role of the three-body force is studied explicitly - namely, it is shown how it enters in the asymptotic normalization coefficient of the wave function. We further verify that this short-range force does not affect the analytic form of the leadingorder volume dependence of the shallow bound state energy. Note that omitting the three-body force altogether renders the three-body problem ill-defined in the infinite volume, since the whole renormalization program fails (see, e.g., refs. [34, 35]). ${ }^{1}$ Hence, its inclusion is a matter of principle and does not simply amount to evaluating corrections to the leading-order result.

(ii) The leading-order result for the finite volume dependence of a shallow three-body bound state is derived for finite scattering length. From the previous derivation in

\footnotetext{
${ }^{1}$ The case of a covariant formulation was recently investigated in ref. [36].
} 
the unitary limit, it is not immediately clear how one can move beyond this approximation, as well as how to build in effective range corrections, mixing with higher partial waves, etc.

(iii) The formalism developed in refs. [22, 23] explicitly excludes poles in the (nonstandard) two-body $K$ matrix. Therefore, it is not obvious how to proceed in the physical situation if deeply-bound dimers exist. ${ }^{2}$ We address this issue and show how the leading-order result for a shallow three-body state goes over into the well-known result for the particle-dimer bound state that can be obtained with the use of the Lüscher equation.

In the present paper, we elaborate on the above issues for a system of three identical bosons. The study of this particularly simple model lays the foundation for the treatment of the general three-body quantization condition in the dimer picture, which is considered in our forthcoming publication [37].

The layout of the paper is follows. In section 2, we consider the role of short-range interactions in the finite-volume behavior of the two-body binding energy. This simple example illustrates the pattern, along which the inclusion of the short-range three-particle force is considered. In section 3, we collect all the information about the particle-dimer formalism in the infinite volume, including the analytic solution of the problem in the unitary limit. The leading order formula for the shallow bound-state energy shift in the unitary limit [19] is rederived in section 4. In addition, we discuss the relation of the asymptotic normalization coefficient in this formula to the short-range three-body force. Section 5 deals with the calculation of the energy shift beyond the unitary limit and the relation to the particle-dimer bound state picture. Finally, section 6 contains our conclusions.

\section{Two-body bound state}

The energy shift of a shallow two-body bound state of identical bosons of mass $m$ in a finite volume is given by the Lüscher formula [38]

$$
\Delta E_{B}=-12 \kappa_{2}\left|\mathcal{A}_{2}\right|^{2} \frac{\exp \left(-\kappa_{2} L\right)}{m L}+\cdots
$$

where $L$ is the size of the cubic box, $\kappa_{2}=\sqrt{m E_{B}}$ is the two-body binding momentum and $\mathcal{A}_{2}$ denotes the two-body asymptotic normalization coefficient (note that we choose a different definition for $\mathcal{A}_{2}$ from that in ref. [38]). The latter is defined through the behavior of the radial bound-state wave function at large distances:

$$
\Psi(r) \sim \sqrt{\frac{\kappa_{2}}{2 \pi}} \mathcal{A}_{2} \frac{\exp \left(-\kappa_{2} r\right)}{r}, \quad \text { as } r \rightarrow \infty .
$$

\footnotetext{
${ }^{2}$ Strictly speaking, the existence of a bound dimer, which is equivalent to the presence of a pole in the $K$-matrix, does not necessarily require the presence of a pole in the non-standard $K$ matrices introduced in refs. [22, 23], and vice versa. The answer to this question may even depend on details of the cutoff used, etc. In our opinion, this situation is not satisfactory and a viable solution requires a formalism that is able to accomodate the presence of a pole in either of these matrices.
} 
Let us derive the relation of the quantity $\mathcal{A}_{2}$ to the parameters characterizing the shortrange interactions. We shall do this using the non-relativistic effective Lagrangians with a method that closely resembles the one used in the three-particle case. Dimensional regularization with minimal subtraction is the most convenient choice, albeit the final results do not depend on the regularization used. This is explicitly demonstrated in appendix A, where we demonstrate that exactly the same results are obtained by using the dimensional regularization with power divergence subtraction. The same applies for the cutoff regularization.

In momentum space, the non-relativistic interaction Hamiltonian consists of an infinite tower of operators with increasing mass dimension. Limiting ourselves to $O\left(\mathbf{p}^{2}\right)$ and to S-waves only, in momentum space we may write

$$
H_{\mathrm{int}}(\mathbf{p}, \mathbf{q}) \doteq\langle\mathbf{p}|V| \mathbf{q}\rangle=2 C_{0}+C_{2}\left(\mathbf{p}^{2}+\mathbf{q}^{2}\right)+O\left(\mathbf{p}^{4}\right),
$$

where the couplings $C_{0}$ and $C_{2}$ specifying the effective potential $V$ are related to the scattering length $a$ and the effective range $r_{e}$ through

$$
C_{0}=\frac{2 \pi a}{m}, \quad C_{2}=\frac{\pi a^{2} r_{e}}{m} .
$$

The bound-state wave function obeys the Schrödinger equation

$$
\left(\mathbf{p}^{2}+\kappa_{2}^{2}\right) \Psi(\mathbf{p})=-m \int \frac{d^{d} \mathbf{q}}{(2 \pi)^{d}} H_{\mathrm{int}}(\mathbf{p}, \mathbf{q}) \Psi(\mathbf{q}),
$$

where $d$ is the dimension of space.

The wave function $\Psi(\mathbf{p})$ consists of a long-range tail and the short-range part, which can be approximated by a polynomial. Consequently, one may try to solve the eq. (2.5) with the following ansatz:

$$
\Psi(\mathbf{p})=\sqrt{8 \pi \kappa_{2}} \frac{\mathcal{A}_{2}}{\mathbf{p}^{2}+\kappa_{2}^{2}}+p_{0}+p_{2} \mathbf{p}^{2}+\cdots .
$$

Substituting this ansatz into the Schödinger equation and performing the integrals in dimensional regularization with minimal subtraction, it is easy to see that $p_{2}=p_{4}=\ldots=0$, while $\mathcal{A}_{2}$ and $p_{0}$ obey the system of linear equations

$$
\begin{aligned}
\sqrt{8 \pi \kappa_{2}} \mathcal{A}_{2}+\kappa_{2}^{2} p_{0} & =\sqrt{8 \pi \kappa_{2}} \mathcal{A}_{2} \frac{\kappa_{2}}{4 \pi}\left(2 m C_{0}-m C_{2} \kappa_{2}^{2}\right), \\
p_{0} & =m C_{2} \sqrt{8 \pi \kappa_{2}} \mathcal{A}_{2} \frac{\kappa_{2}}{4 \pi} .
\end{aligned}
$$

Substituting the second equation into the first one, an equation to determine the bound state momentum $\kappa_{2}$ emerges

$$
1-\frac{\kappa_{2} m}{2 \pi}\left(C_{0}-C_{2} \kappa_{2}^{2}\right)=0 .
$$

The system of the homogeneous linear equations, eq. (2.7) determines only the ratio $p_{0} / \mathcal{A}_{2}$. In order to determine $\mathcal{A}_{2}$, one uses the normalization condition for the wave function

$$
\int \frac{d^{d} \mathbf{q}}{(2 \pi)^{d}}|\Psi(\mathbf{q})|^{2}=\int \frac{d^{d} \mathbf{q}}{(2 \pi)^{d}}\left(\sqrt{8 \pi \kappa_{2}} \frac{\mathcal{A}_{2}}{\mathbf{p}^{2}+\kappa_{2}^{2}}+p_{0}\right)^{2}=1 .
$$


Evaluating the integrals and expressing $p_{0}$ through $\mathcal{A}_{2}$, we finally get ${ }^{3}$

$$
\mathcal{A}_{2}^{2}=\left(1-\frac{m C_{2} \kappa_{2}^{3}}{\pi}\right)^{-1}=\frac{1}{1-\kappa_{2} r_{e}}
$$

where at the final stage we have expressed the non-relativistic couplings through the physical observables.

Below, we briefly summarize the lessons learned:

(i) The energy level shift in a finite volume is determined by the asymptotic part of the bound state wave function, which is parameterized by two constants $\kappa_{2}$ and $\mathcal{A}_{2}$.

(ii) If the higher-order short-range interactions are absent $\left(r_{e}=0\right.$ together with all higher-order terms), then $\mathcal{A}_{2}=1$. This condition is equivalent to Weinberg's compositeness condition [40], which distinguishes a hadronic molecule from a tightly bound compound.

(iii) The asymptotic normalization coefficient is determined from the normalization of the whole wave function to unity. For example, $\mathcal{A}_{2} \simeq 0$ would mean that the short-range component prevails over the long-range one, so that the system is predominately a tight compound. In accordance with this, the energy level has very little dependence on the volume, and vice versa.

In the next section, we demonstrate, how the above derivation can be extended to the particle-dimer bound state.

\section{Dimer formalism in the infinite volume}

\subsection{The Lagrangian}

In order to simplify things as much as possible, we shall consider the case of three identical non-relativistic bosons in the CM frame. The inclusion of relativistic kinematics, spin, moving frames, etc., proceeds along the standard path and will be addressed in our future publications.

In the following, we shall mainly follow the refs. [34, 35]. The most general effective Lagrangian that describes the two- and three-particle sectors is given by

$$
\mathcal{L}=\psi^{\dagger}\left(i \partial_{0}+\frac{\nabla^{2}}{2 m}\right) \psi-\frac{C_{0}}{2}\left(\psi^{\dagger} \psi\right)^{2}-\frac{D_{0}}{6}\left(\psi^{\dagger} \psi\right)^{3}+\cdots,
$$

where $\psi$ denotes the non-relativistic field operator for a boson with a mass $m$, and ellipses stand for the terms with derivatives. We further introduce a dummy field $T$ (called dimer) with the quantum number of two bosons and consider the Lagrangian

$$
\mathcal{L}=\psi^{\dagger}\left(i \partial_{0}+\frac{\nabla^{2}}{2 m}\right) \psi+\Delta T^{\dagger} T-\frac{g}{\sqrt{2}}\left(T^{\dagger} \psi \psi+\text { h.c. }\right)+h T^{\dagger} T \psi^{\dagger} \psi+\cdots
$$

\footnotetext{
${ }^{3} \mathrm{~A}$ general formula, valid to all orders in the momentum expansion, can be found, e.g., in ref. [39]: $\mathcal{A}_{2}^{2}=\left(1+2 \kappa_{2} D\right)^{-1}$, where $D=\left.\frac{d}{d k^{2}}(k \cot \delta)\right|_{k=i \kappa_{2}}$.
} 
Note that the field $T$ is not dynamical - the corresponding Lagrangian does not contain the time derivative. Integrating out this field by using the equations of motion, we arrive at the Lagrangian

$$
\mathcal{L}=\psi^{\dagger}\left(i \partial_{0}+\frac{\nabla^{2}}{2 m}\right) \psi-\frac{g^{2}\left(\psi^{\dagger} \psi\right)^{2}}{2\left(\Delta+h \psi^{\dagger} \psi\right)}+\cdots
$$

Expanding this Lagrangian in the power of fields, one sees that it describes exactly the same physics as the Lagrangian from eq. (3.1) in the two- and three-particle sectors, if the couplings are fixed in the following manner:

$$
C_{0}=\frac{g^{2}}{\Delta}, \quad D_{0}=-\frac{3 g^{2} h}{\Delta^{2}}
$$

The following remarks are in order:

(i) The particle-dimer picture is not an approximation, in fact, being restricted to the two and three particle sectors, it is mathematically equivalent to the original treatment without a dimer field (for more discussion, see ref. [37]). Hence, the treatment of the finite-volume effects with the use of the particle-dimer approach is as general as the one based on a three-particle Lagrangian without a dimer field.

(ii) Using the dimer formalism does not imply the neglect of the higher partial waves. The two-particle Lagrangian containing derivative terms, which describe P-, D-, ... wave interactions, can be replaced by a tower of Lagrangians containing dimers with angular momentum 1, 2, etc... The truncation of the partial-wave expansion is then equivalent to including the dimers with angular momentum below some fixed value (the details of the formalism can be found in ref. [37]).

One additional remark concerns the inclusion of the kinetic energy term for the dimer. In principle, $T$ is a dummy field, so, instead of eq. (3.2), one could consider the Lagrangian with a dynamical dimer field as well

$$
\mathcal{L}=\psi^{\dagger}\left(i \partial_{0}+\frac{\nabla^{2}}{2 m}\right) \psi+\sigma T^{\dagger}\left(i \partial_{0}+\frac{\nabla^{2}}{4 m}-\Delta\right) T-\frac{g}{\sqrt{2}}\left(T^{\dagger} \psi \psi+\text { h.c. }\right)+h T^{\dagger} T \psi^{\dagger} \psi+\cdots
$$

where $\sigma= \pm 1$ is sign that depends on the sign of the effective range. The variable $T$ can again be integrated out, leading to an equivalent theory in terms of a field $\psi$ only. ${ }^{4}$

In the following, we shall use the formulation based on the Lagrangian, eq. (3.2), neglecting all higher-order terms. The inclusion of the derivative couplings, higher partial waves, etc. will be discussed in our forthcoming paper.

\footnotetext{
${ }^{4}$ We note, as argued, e.g., in ref. [41], that when a shallow two-particle bound state is present, the convergence radius of the perturbation expansion in the theory with a dynamical dimer should be larger because this theory contains the small scale $\Delta$ explicitly (i.e., not hidden in the couplings of the effective theory).
} 


\subsection{The bound-state equation and the normalization condition}

As is well known, the particle-dimer bound state wave function in the theory described by the Lagrangian, eq. (3.2), obeys the homogeneous Faddeev equation (see, e.g., refs. [34, 35])

$$
\Psi(\mathbf{p})=8 \pi \int^{\Lambda} \frac{d^{3} \mathbf{q}}{(2 \pi)^{3}} Z(\mathbf{p}, \mathbf{q} ; E) \tau(\mathbf{q} ; E) \Psi(\mathbf{q}),
$$

where $\Lambda$ denotes an explicit UV cutoff, and

$$
\begin{aligned}
Z(\mathbf{p}, \mathbf{q} ; E) & =\frac{1}{-m E+\mathbf{p}^{2}+\mathbf{q}^{2}+\mathbf{p q}}+\frac{h}{2 m g^{2}}, \\
\tau(\mathbf{q} ; E) & =\frac{1}{-a^{-1}+\sqrt{\frac{3}{4} \mathbf{q}^{2}-m E}}
\end{aligned}
$$

Projecting to the S-wave and defining $h=2 m g^{2} H(\Lambda) / \Lambda^{2}, \kappa^{2}=-m E$, we arrive at the equation

$$
\Psi(p)=\frac{4}{\pi} \int_{0}^{\Lambda} q^{2} d q\left\{\frac{1}{2 p q} \ln \frac{p^{2}+p q+q^{2}+\kappa^{2}}{p^{2}-p q+q^{2}+\kappa^{2}}+\frac{H(\Lambda)}{\Lambda^{2}}\right\} \tau(q ; E) \Psi(q),
$$

where $\Psi(p)$ stands for the S-wave wave function (note that $\tau(\mathbf{q} ; E)=\tau(q ; E)$ depends only on $q=|\mathbf{q}|)$. It is well known that the particle-dimer coupling constant $H(\Lambda)$ should be a $\log$-periodic function of the cutoff parameter $\Lambda$ for the limit $\Lambda \rightarrow \infty$ to exist in this equation $[34,35]$.

Next, we shall derive the normalization condition for the wave function $\Psi(p)$, which has a non-trivial form because the kernel of the integral equation depends on the energy $E$. The derivation follows the standard pattern (see, e.g., [42]). Namely, we consider the inhomogeneous equation for the scattering amplitude

$$
\mathcal{M}(\mathbf{p}, \mathbf{k} ; E)=Z(\mathbf{p}, \mathbf{k} ; E)+8 \pi \int^{\Lambda} \frac{d^{3} \mathbf{q}}{(2 \pi)^{3}} Z(\mathbf{p}, \mathbf{q} ; E) \tau(\mathbf{q} ; E) \mathcal{M}(\mathbf{q}, \mathbf{k} ; E) .
$$

In a compact notation, we have $\mathcal{M}=Z+Z(8 \pi \tau) \mathcal{M}$. Defining the Green function as $G=(8 \pi \tau)+(8 \pi \tau) \mathcal{M}(8 \pi \tau)$, we obtain $G=(8 \pi \tau)+(8 \pi \tau) Z G$, and $G^{-1}=(8 \pi \tau)^{-1}-Z$. Further, using the identity $G G^{-1} G=G$ and the behavior of the Green function in the vicinity of the bound-state pole

$$
G(\mathbf{p}, \mathbf{k} ; E)=\frac{8 \pi \tau\left(\mathbf{p} ; E_{n}\right) \Psi_{n}(\mathbf{p}) \Psi_{n}(\mathbf{k}) 8 \pi \tau\left(\mathbf{k} ; E_{n}\right)}{E-E_{n}}+\text { terms regular as } E \rightarrow E_{n},
$$

we arrive at the following normalization condition for the wave function:

$$
\begin{aligned}
1= & -\left.8 \pi \int^{\Lambda} \frac{d^{3} \mathbf{p}}{(2 \pi)^{3}}\left(\Psi_{n}(\mathbf{p})\right)^{2} \frac{\partial \tau(\mathbf{p} ; E)}{\partial E}\right|_{E=E_{n}} \\
& -\left.(8 \pi)^{2} \int^{\Lambda} \frac{d^{3} \mathbf{p}}{(2 \pi)^{3}} \frac{d^{3} \mathbf{k}}{(2 \pi)^{3}} \Psi_{n}(\mathbf{p}) \tau(\mathbf{p} ; E) \frac{\partial Z(\mathbf{p}, \mathbf{k} ; E)}{\partial E} \tau(\mathbf{k} ; E) \Psi_{n}(\mathbf{k})\right|_{E=E_{n}} .
\end{aligned}
$$




\subsection{Minlos-Faddeev solution}

Assuming $\Lambda \rightarrow \infty$ and $H(\Lambda)=0$, we obtain the Skornyakov-Ter-Martirosian (STM) equation [43]. Unlike the equation (3.6), the STM equation is known not to possess a unique solution [44]. Minlos and Faddeev [45] have found an exact solution to the integral equation in the unitary limit $a \rightarrow \infty$ :

$$
\Psi_{0}\left(\frac{p}{\kappa}\right)=i N_{0} \frac{\kappa \sin \left(s_{0} u\right)}{p}, \quad u=\ln \left(\frac{\sqrt{3}}{2} \frac{p}{\kappa}+\sqrt{\frac{3 p^{2}}{4 \kappa^{2}}+1}\right)
$$

where $\kappa=\sqrt{-m E}$ is the three-body bound state momentum and $s_{0} \simeq 1.00624$ is a numerical constant, which is a solution of the transcendental equation

$$
s_{0} \cosh \frac{\pi s_{0}}{2}=\frac{8}{\sqrt{3}} \sinh \frac{\pi s_{0}}{6} .
$$

We hereafter refer to eq. (3.12) as to the Minlos-Faddeev (MF) wave function. Note that the function in eq. (3.12) is a solution for any value of $\kappa$ - the spectrum is not quantized. The overall normalization factor in this equation should be determined from the normalization condition. The substitution of eq. (3.12) into (3.11) gives

$$
\frac{\pi}{2 m N_{0}^{2}}=I_{0},
$$

where (see appendix B)

$$
\begin{aligned}
I_{0} & =\int_{0}^{\infty} d x \frac{\sin ^{2}\left(s_{0} u\right)}{\left(\sqrt{\frac{3 x^{2}}{4}+1}\right)^{3}}+\frac{8}{\pi} \int_{0}^{\infty} \frac{x d x}{\sqrt{\frac{3 x^{2}}{4}+1}} \int_{0}^{\infty} \frac{y d y}{\sqrt{\frac{3 y^{2}}{4}+1}} \frac{\sin \left(s_{0} u\right) \sin \left(s_{0} v\right)}{\left(x^{2}+y^{2}+1\right)^{2}-x^{2} y^{2}} \\
& =\frac{1}{\sqrt{3}}\left(1-\frac{\pi s_{0}}{\sinh \pi s_{0}}\right)+\frac{8 \pi}{9 \sinh \pi s_{0}}\left(\sinh \frac{2 \pi s_{0}}{3}-2 \sinh \frac{\pi s_{0}}{3}\right)
\end{aligned}
$$

and

$$
u=\ln \left(\frac{\sqrt{3}}{2} x+\sqrt{\frac{3}{4} x^{2}+1}\right), \quad v=\ln \left(\frac{\sqrt{3}}{2} y+\sqrt{\frac{3}{4} y^{2}+1}\right)
$$

\subsection{Asymptotic normalization coefficient}

As mentioned above, the STM equation does not have unique solutions. First, imposing a cutoff, one arrives at the discrete three-particle spectrum. In order to ensure that the limit $\Lambda \rightarrow \infty$ exists, one has to introduce a short-range interaction parameterized by a constant $H(\Lambda)$, where the dependence on the cutoff $\Lambda$ is log-periodic. Furthermore, for a fixed $\Lambda$ and $H(\Lambda)$, the low-energy spectrum is discrete, condensing towards zero. For two neighboring levels from this tower of so-called Efimov states [46-48], whose energy is much smaller than the cutoff $\Lambda$, the following relation holds in the unitary limit:

$$
\frac{\kappa_{n+1}}{\kappa_{n}}=\exp \left(-\pi / s_{0}\right) \simeq \frac{1}{22.69} .
$$




\begin{tabular}{|c|c|c|}
\hline$n$ & $\kappa_{n}$ & $\kappa_{n+1} / \kappa_{n}$ \\
\hline 1 & 1779.3756 & 22.93 \\
2 & 77.5971 & 22.69 \\
3 & 3.4192 & 22.69 \\
4 & 0.1507 & 22.69 \\
5 & 0.006639 & \\
& $\ldots$ & \\
\hline
\end{tabular}

Table 1. Some energy levels obtained eq. (3.8) for the choice $\Lambda=10^{4}$ and $H(\Lambda)=0$.

Consequently, fixing a single energy level for a given $\Lambda$ is equivalent to the fixing of the parameter $H(\Lambda)$.

The Faddeev equation with a finite cutoff and $H(\Lambda)$ can not be solved analytically. However, the numerical solution is straightforward. In table 1, for illustration, we give several energy eigenvalues for the choice $\Lambda=10^{4}$ and $H(\Lambda)=0$.

It is clear that, for the momenta much smaller than the cutoff $\Lambda$, the wave function will be given by the MF solution $\Psi_{0}$. The difference can arise only at momenta $p \simeq \Lambda$. The overall normalization, however, is a subtler issue since the normalization integral includes all momenta. To summarize, the solution of the Faddeev equation with cutoff at a given bound-state momentum $\kappa$ should be given by

$$
\Psi\left(\frac{p}{\kappa}\right)=A\left(\frac{p}{\kappa}\right) \Psi_{0}\left(\frac{p}{\kappa}\right)
$$

where the function $A(x)$ should have a very flat plateau for $x \ll \Lambda / \kappa$. Then, in analogy to the two-body case, we define the particle-dimer asymptotic normalization coefficient as

$$
\mathcal{A}=A(0)
$$

Further, the dimensionless quantity $\mathcal{A}$ must be a function of the only dimensionless combination $\kappa / \Lambda$ that can be composed from the parameters of the theory. If $\kappa / \Lambda \rightarrow 0$, then, obviously, $\mathcal{A} \rightarrow 1$. Consequently, the asymptotic normalization coefficient is very close to one for the shallow three-particle bound states. The explicit demonstration of the above statements is given in figure 1.

The situation changes, when derivative particle-dimer interactions are added. Consider, for example, adding the term $H_{1}(\Lambda)\left(\mathbf{p}^{2}+\mathbf{q}^{2}\right)$ to the particle-dimer interaction Hamiltonian (this is an analog of the effective range term in the two-particle case). The ratio will be still flat for $p \ll \Lambda$. However, the statement $\mathcal{A} \rightarrow 1$, as $\kappa / \Lambda \rightarrow 0$ does not hold any more - in other words, the asymptotic normalization coefficient encodes the effect of the short-range physics, as in the two-particle case. In the particle-dimer case, however, $\mathcal{A}$ and $H_{1}(\Lambda)$ can not be related algebraically, and $\mathcal{A}$ should be extracted from the numerical solution of the equation with a given value of $H_{1}(\Lambda) \neq 0$. 


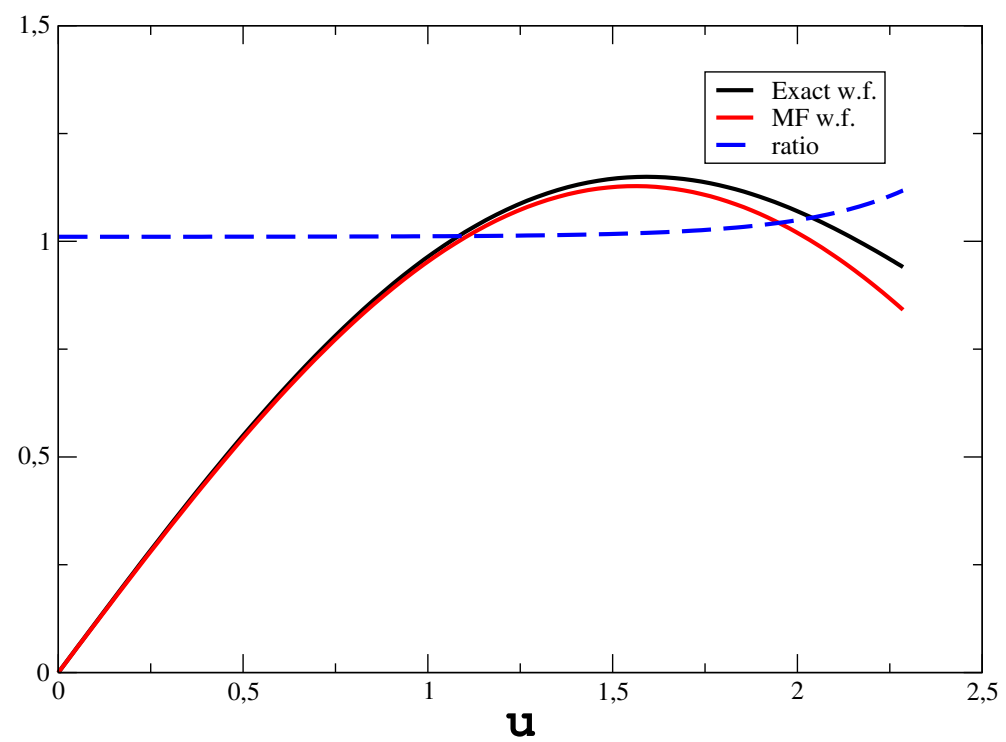

Figure 1. The numerical solution of the Faddeev equation vs. the Minlos-Faddeev wave function. The parameters are taken as $\Lambda=10^{4}$ and $\kappa=1779.3756$, corresponding to $H(\Lambda)=0$. We namely plot the functions $|p / \kappa \Psi(p / \kappa)|,\left|p / \kappa \Psi_{0}(p / \kappa)\right|$ and $A(p / \kappa)$ from eq. (3.18) vs. the dimensionless variable $u$, which is defined in eq. (3.12). The ratio of two functions, $\mathcal{A}$, is very flat and approaches the value $\mathcal{A}=1.0105863$ at the origin.

\section{Particle-dimer bound state in a finite volume}

\subsection{Expression of the first order finite-volume energy shift}

Our derivation — in the particle-dimer context — will be partly similar to that of ref. [27]. Consider the Faddeev equation in a finite volume

$$
\mathcal{M}_{L}(\mathbf{p}, \mathbf{k} ; E)=Z(\mathbf{p}, \mathbf{k} ; E)+\frac{8 \pi}{L^{3}} \sum_{\mathbf{q}} Z(\mathbf{p}, \mathbf{q} ; E) \tau_{L}(\mathbf{q} ; E) \mathcal{M}_{L}(\mathbf{q}, \mathbf{k} ; E)
$$

where $\mathbf{q}=\frac{2 \pi}{L} \mathbf{n}, \quad \mathbf{n} \in \mathbb{Z}^{3}$ and

$$
\begin{aligned}
\tau_{L}^{-1}(\mathbf{q} ; E) & =-a^{-1}+\sqrt{\frac{3}{4} \mathbf{q}^{2}+\kappa_{L}^{2}}+\Delta_{L}(\mathbf{q} ; E), \\
\Delta_{L}(\mathbf{q} ; E) & =-\frac{1}{\pi L} \int d^{3} \mathbf{s} \sum_{\mathbf{n} \neq \mathbf{0}} \frac{e^{2 \pi i \mathbf{n} \mathbf{s}-i \pi \hat{\mathbf{q}} \mathbf{n}}}{\hat{\kappa}_{L}^{2}+\frac{3}{4} \hat{\mathbf{q}}^{2}+\mathbf{s}^{2}}, \quad \hat{\mathbf{q}}=\frac{L \mathbf{q}}{2 \pi}, \quad \hat{\kappa}_{L}=\frac{L \kappa_{L}}{2 \pi} .
\end{aligned}
$$

Here, $\kappa_{L}^{2}=-m E$. Moreover, we have assumed that we are below the particle-dimer breakup threshold, where all denominators are non-singular, and have used Poisson's summation formula. Note that exactly this equation was considered earlier in refs. [30-33].

The finite-volume effects in the particle-dimer bound state equation emerge at two different places. First, the integration over $\mathbf{q}$ is changed to a sum over discrete values in eq. (4.1). Second, there is an additional term $\Delta_{L}(\mathbf{q} ; E)$ in eq. (4.2). Using again Poisson's 
summation formula, we may rewrite the eq. (4.1) as

$$
\begin{aligned}
\mathcal{M}_{L}(\mathbf{p}, \mathbf{k} ; E) & =Z(\mathbf{p}, \mathbf{k} ; E)+8 \pi \int^{\Lambda} \frac{d^{3} \mathbf{q}}{(2 \pi)^{3}} Z(\mathbf{p}, \mathbf{q} ; E) \hat{\tau}_{L}(\mathbf{q} ; E) \mathcal{M}_{L}(\mathbf{q}, \mathbf{k} ; E), \\
\hat{\tau}_{L}(\mathbf{q} ; E) & =\frac{1+\sum_{\mathbf{n} \neq \mathbf{0}} e^{2 i \pi \mathbf{n} \hat{\mathbf{q}}}}{\tau^{-1}(\mathbf{q} ; E)+\Delta_{L}(\mathbf{q} ; E)} .
\end{aligned}
$$

Eliminating now the quantity $Z$ using eq. (3.9), we obtain

$$
\mathcal{M}_{L}(\mathbf{p}, \mathbf{k} ; E)=\mathcal{M}(\mathbf{p}, \mathbf{k} ; E)+8 \pi \int^{\Lambda} \frac{d^{3} \mathbf{q}}{(2 \pi)^{3}} \mathcal{M}(\mathbf{p}, \mathbf{k} ; E) \delta \tau_{L}(\mathbf{q} ; E) \mathcal{M}_{L}(\mathbf{q}, \mathbf{k} ; E),
$$

where

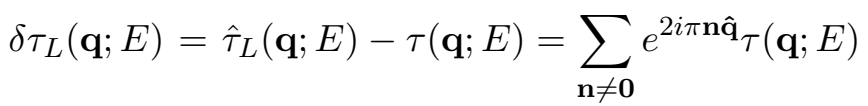

$$
\begin{aligned}
& -\left(1+\sum_{\mathbf{n} \neq \mathbf{0}} e^{2 i \pi \mathbf{n} \hat{\mathbf{q}}}\right)(\tau(\mathbf{q} ; E))^{2} \Delta_{L}(\mathbf{q} ; E)+\cdots .
\end{aligned}
$$

The infinite-volume amplitude $\mathcal{M}$ has a pole at the bound-state energy (cf. with Eq, (3.10)):

$$
\mathcal{M}(\mathbf{p}, \mathbf{k} ; E)=\frac{\Psi(\mathbf{p}) \Psi(\mathbf{k})}{E-E_{L}}+\text { terms regular as } E \rightarrow E_{L},
$$

where $\Psi(\mathbf{p})$ is the infinite-volume wave function. Substituting this ansatz in eq. (4.4), we finally obtain the expression for the first-order energy shift of the three-body bound state

$$
\Delta E_{L}=8 \pi \int^{\Lambda} \frac{d^{3} \mathbf{p}}{(2 \pi)^{3}}(\Psi(\mathbf{p}))^{2} \delta \tau_{L}(\mathbf{p} ; E) .
$$

\subsection{Evaluation of the first-order energy shift}

The energy shift can be written as

$$
\begin{aligned}
& \Delta E_{L}=\Delta E_{1}+\Delta E_{2}+\cdots, \\
& \Delta E_{1}=8 \pi \int^{\Lambda} \frac{d^{3} \mathbf{p}}{(2 \pi)^{3}} \frac{(\Psi(\mathbf{p}))^{2} \sum_{\mathbf{n} \neq \mathbf{0}} e^{2 i \pi \mathbf{n} \hat{\mathbf{p}}}}{-a^{-1}+\sqrt{\frac{3}{4} \mathbf{p}^{2}+\kappa^{2}}}, \\
& \Delta E_{2}=-8 \pi \int^{\Lambda} \frac{d^{3} \mathbf{p}}{(2 \pi)^{3}} \frac{(\Psi(\mathbf{p}))^{2}\left(1+\sum_{\mathbf{n} \neq \mathbf{0}} e^{2 i \pi \mathbf{n} \hat{\mathbf{p}}}\right)}{\left(-a^{-1}+\sqrt{\frac{3}{4} \mathbf{p}^{2}+\kappa^{2}}\right)^{2}} \Delta_{L}(\mathbf{p}, E) .
\end{aligned}
$$

The evaluation of these integrals in the unitary limit proceeds mainly along the lines described in ref. [27]. In this limit, one has $\Lambda \rightarrow \infty, a \rightarrow \infty$ and $\Psi(\mathbf{p})$ is the MF wave function $\Psi_{0}(\mathbf{p})$. Using eq. (3.12) and performing angular integration, we get

$$
\Delta E_{1}=-\frac{2 N_{0}^{2} \kappa^{2}}{\pi} \int_{0}^{\infty} d p \sin ^{2}\left(s_{0} \ln \left(\frac{\sqrt{3}}{2} \frac{p}{\kappa}+\sqrt{\frac{3 p^{2}}{4 \kappa^{2}}+1}\right)\right) \sum_{\mathbf{n} \neq \mathbf{0}} \frac{e^{i L n p}-e^{-i L n p}}{i L n p} \frac{1}{\sqrt{\frac{3 p^{2}}{4}+\kappa^{2}}},
$$


where $n=|\mathbf{n}|$. It is clear that the leading exponential contribution emerges from the term with $n=1$. Introducing the variable $u$ defined in eq. (3.12), one gets

$$
\frac{m \Delta E_{1}}{\kappa^{2}}=-\frac{6}{\kappa L I_{0}} \int_{0}^{\infty} \frac{d u}{\sinh u}\left(1-\cos 2 s_{0} u\right) \sin \left(\frac{2 \kappa L}{\sqrt{3}} \sinh u\right) .
$$

in $\kappa L \gg 1$, the integral in the r.h.s. of the above equation has the following asymptotic expansion

$$
\int_{0}^{\infty} \frac{d u}{\sinh u}\left(1-\cos 2 s_{0} u\right) \sin \left(\frac{2 \kappa L}{\sqrt{3}} \sinh u\right)=-\frac{3^{1 / 4} \pi^{1 / 2}}{2 \sqrt{\kappa L}}\left(1-\cosh \pi s_{0}\right) \exp \left(-\frac{2 \kappa L}{\sqrt{3}}\right)+\cdots
$$

Using this expansion, one reproduces the result first derived in ref. [19] and re-derived in ref. [27] (note that in ref. [27], an algebraic error contained in the original derivation was corrected):

$$
\frac{\Delta E_{1}}{|E|}=c(\kappa L)^{-3 / 2} \exp \left(-\frac{2 \kappa L}{\sqrt{3}}\right)+\cdots
$$

where

$$
c=-\frac{2 \pi^{1 / 2} 3^{5 / 4}}{I_{0}} \sinh ^{2} \frac{\pi s_{0}}{2} .
$$

Taking into account the relation

$$
I_{0}=\frac{C_{0}^{-1}}{6 \sqrt{3} \pi^{3}}
$$

where $C_{0}^{-1}$ is defined in eq. (16) of ref. [19], it is straightforward to verify that eq. (4.12) is identical to the final result of ref. [19]..$^{5}$ However, eq. (4.12) contains more information than the original formula from ref. [19]. It corresponds to the unit asymptotic normalization coefficient $\mathcal{A}=1$. Now, it is clear, where the non-trivial three-particle force, encoded in the derivative particle-dimer couplings, will reveal itself: the $L$-dependence in the formula (4.12) remains the same, only the overall factor will be multiplied by $\mathcal{A}^{2} \neq 1$, where $\mathcal{A}$ can be determined from the infinite-volume solution through the procedure described above. The reason for this is that, at small momenta $p \ll \Lambda$, the ratio $A$ defined in eq. (3.18) is close to constant and does not affect the large- $L$ behavior of the energy level.

Further, as shown in ref. [27], the correction $\Delta E_{2}$ is subleading and behaves as

$$
\Delta E_{2} \propto(\kappa L)^{-5 / 2} \exp \left(-\frac{2 \kappa L}{\sqrt{3}}\right)
$$

for a large $L$. The subsequent terms are even more suppressed.

To summarize, we have reproduced the result of ref. [19] for the leading finite-volume energy shift of the three-body bound state in the unitary limit in the particle-dimer picture. Moreover, we have shown that in the unitary limit, the asymptotic normalization coefficient emerges from three-particle derivative forces, and this coefficient is equal to one if such forces are absent.

At the next step, we investigate the system beyond the unitary limit.

\footnotetext{
${ }^{5}$ Note that the $C_{0}^{-1}$ defined in eq. (16) of ref. [19] is not related to the $C_{0}$ defined in eq. (2.3).
} 


\section{Beyond the unitary limit}

As seen, the correction $\Delta E_{1}$ given by eq. (4.8), gives the leading contribution to the finitevolume energy level in the unitary limit. We expect that this statement stays valid for finite values of $a$ (see the discussion below). Singling out the contribution with $|\mathbf{n}|=1$, one may rewrite the leading contribution to $\Delta E$ in the following form

$$
\Delta E \propto \int^{\Lambda} \frac{d^{3} \mathbf{p}}{(2 \pi)^{3}} \frac{(\Psi(\mathbf{p}))^{2} e^{2 i \pi \mathbf{n} \hat{\mathbf{p}}}}{-a^{-1}+\sqrt{\frac{3}{4} \mathbf{p}^{2}+\kappa^{2}}}+\cdots, \quad|\mathbf{n}|=1 .
$$

As we demonstrate in appendix $\mathrm{C}$, the wave function $\Psi(\mathbf{p})$ is regular near the origin (more precisely, the singularities of $\Psi(\mathbf{p})$ are located much farther from the origin than the singularities of the denominator). This means that the singularities of $\Psi(\mathbf{p})$ do not contribute to the large- $L$ behavior of the energy shift at leading order and hence, at this order, $\Psi(\mathbf{p})$ can be replaced by a constant. Performing the angular integration, we arrive at the following result

$$
\Delta E \propto \frac{1}{L} \int_{-\infty}^{\infty} \frac{p d p}{2 \pi i} \frac{e^{i p L}\left(a^{-1}+\sqrt{\frac{3}{4} \mathbf{p}^{2}+\kappa^{2}}\right)}{\frac{3}{4} \mathbf{p}^{2}+\kappa^{2}-a^{-2}} .
$$

Note that the quantity $\kappa^{2}-a^{-2}$ is always positive, if a bound state of a particle and a bound dimer is considered (recall that $\kappa_{2}=a^{-1}$ is the binding momentum of the dimer in the unitary limit). One has to distinguish two limiting cases:

\subsection{A shallow bound state of a particle and a deeply bound dimer}

In this case, we have $\kappa^{2}-a^{-2} \ll \kappa^{2}$. The singularity at $p= \pm i \sqrt{\frac{4}{3}\left(\kappa^{2}-a^{-2}\right)}$ is dominant, and the singularity arising from the square root (cut) can be neglected. Performing the Cauchy integration, we get

$$
\Delta E \propto \frac{1}{L} \exp \left(-\frac{2}{\sqrt{3}} \sqrt{\kappa^{2}-a^{-2}} L\right)
$$

In other words, we reproduce Lüscher's original result for a two particle (particle-dimer) bound state [38]. Note also that this is in a complete agreement with the result of the recent paper [49].

\subsection{A shallow bound state of three particles}

This corresponds to the opposite limit $\kappa^{2} \gg a^{-2}$. Then, the first term in eq. (5.2) is very small and the energy shift is dominated by the second term. It is straightforward to see that, in this case,

$$
\Delta E \propto \frac{1}{L^{3 / 2}} \exp \left(-\frac{2}{\sqrt{3}} \kappa L\right) .
$$

In other words, the result of ref. [19] is reproduced in this limit. To be more precise, for any small but finite value of $a^{-2}$ the asymptotic behavior of the energy shift is still given by 
eq. (5.3). However, the coefficient of the leading term is very small, whereas the coefficient in front of the subleading term given by eq. (5.4) is of order of unity. So, for large (but not asymptotically large) values of $L$ the behavior is given by eq. (5.4), whereas eq. (5.3) sets in asymptotically.

\subsection{Subleading contributions}

Finally, let us give the arguments in favor of the statement that $\Delta E_{1}$ stays the leading correction beyond the unitary limit. To this end, one has to consider the finite-volume correction, coming from the self-energy of the dimer. To the leading order, this correction is given by the quantity $\Delta E_{2}$ in eq. (4.8). In the unitary limit, it is $O\left(L^{-1}\right)$ suppressed as compared to $\Delta E_{1}$, see ref. [27]. This can be seen, e.g., from eq. (A.11) of that paper after using the method of the steepest descent. Beyond the unitary limit, the denominator $1+3 \ell^{2} / 3 \kappa^{2}$ in that equation gets replaced by $\left(a^{-1}-\sqrt{1+3 \ell^{2} / 3 \kappa^{2}}\right)^{2}$ (cf. with eq. (4.8) from the present paper, where the wave function can be replaced by a constant). It is straightforward to see that this contribution still vanishes as $L^{-5 / 2}$, multiplied by an exponential.

\section{Conclusions}

Our conclusions are as follows:

(i) In this paper, we have rederived the well-known result [19] for the leading-order finite volume energy shift of a shallow three-particle bound state in the unitary limit using the dimer formalism. While this result was not unexpected, since the particle-dimer picture is algebraically equivalent to the three-particle description, it provides a useful check on the particle-dimer formalism in a finite volume.

(ii) Our treatment goes beyond refs. [19, 27]. Namely, we explicitly concentrate on the role of the three-particle force, which is necessary to carry out the renormalization program in the infinite volume. We have verified that the algebraic form of the leading-order formula does not change in the presence of the three-particle force, and only the numerical value of the particle-dimer asymptotic normalization constant is altered (in ref. [27], the three-particle force is included from the beginning, but its contribution to the asymptotic normalization coefficient is not studied). This constant is equal to one for the STM equation and differs from unity in the presence of derivative three-particle interactions - similar to the two-body case.

(iii) Finally, we go beyond the unitary limit and derive the leading-order formula in this case. This formula smoothly interpolates between two extremes: the well-known three-particle bound state in the unitary limit and the bound state of a particle and a deeply bound dimer, for which the usual Lüscher formula applies. The study of these limits enables us to explore the region of applicability of the energy shift formula from ref. [19].

(iv) A host of additional effects awaits to be included, namely, the effective range expansion in the two particle sector, higher partial waves and partial wave mixing, non-rest 
frames, relativistic kinematics, etc. Moreover, a general and tractable quantization condition, which could be used by lattice practitioners to analyze the data in the three-particle sector, remains to be worked out. The particle-dimer language allow one to achieve most of the above goals with an impressive ease. However, we relegate the proof of this statement to our forthcoming publication [37].

\section{Acknowledgments}

The authors would like to thank R. Briceno, Z. Davoudi, M. Döring, E. Epelbaum, M. Hansen, D. Lee, T. Luu, M. Mai, U.-G. Meißner and S. Sharpe for useful discussions and D. Lee and U.-G. Meißner for comments on the manuscript.

We acknowledge support from the DFG through funds provided to the Sino-German CRC 110 "Symmetries and the Emergence of Structure in QCD" and the CRC 1245 "Nuclei: From Fundamental Interactions to Structure and Stars" as well as the BMBF under contract 05P15RDFN1. This research is also supported in part by Volkswagenstiftung under contract no. 86260 and by Shota Rustaveli National Science Foundation (SRNSF), grant no. DI-2016-26.

\section{A Two-body problem using power divergence subtraction}

In section 2, we have discussed the energy level shift of a shallow two-body bound state and showed that the whole effect of the short-range interactions is concentrated in the twobody asymptotic normalization coefficient $\mathcal{A}_{2}$. Dimensional regularization with minimal subtraction was used in the derivation.

In this appendix, we demonstrate that the same result is obtained in dimensional regularization with power divergence subtraction [50], where poles in $1 /(d-3)$ and in $1 /(d-2)$ are subtracted from the integrals ( $d$ is the number of spatial dimensions). This generates a non-trivial dependence of the couplings $C_{0}$ and $C_{2}$ on the renormalization scale $\mu$ which must cancel in physical observables. ${ }^{6}$

The Schrödinger equation for the wave function in the S-wave is rewritten as

$$
\left(p^{2}+\kappa_{2}^{2}\right) \Psi(p)=-m \int \frac{d^{d} \mathbf{q}}{(2 \pi)^{d}} H_{\mathrm{int}}(p, q) \Psi(q), \quad H(p, q)=2 C_{0}(\mu)+C_{2}(\mu)\left(p^{2}+q^{2}\right)+\ldots
$$

Here, $\mu$ denotes the renormalization scale. The coupling constants $C_{0}(\mu)$ and $C_{2}(\mu)$ can be determined from matching to the effective range expansion for the two-body scattering amplitude. We obtain

$$
C_{0}(\mu)=\frac{2 \pi}{m}\left(\frac{1}{a}-\mu\right)^{-1}, \quad C_{2}(\mu)=\frac{m}{2 \pi} C_{0}(\mu)^{2} \frac{r_{e}}{2},
$$

where $|a| \gg r_{e}$ was assumed.

\footnotetext{
${ }^{6}$ We have also checked that the same result is obtained when divergent integrals are regularized with a momentum cutoff $\Lambda$ but we refrain from showing explicit expressions here. In this case, divergences up to fifth order in the cutoff $\Lambda$ appear. Moreover, lower-order couplings are renormalized by higher orders, which leads to more complicated equations.
} 
Using again the ansatz from eq. (2.6) with $p_{2}=\ldots=0$, we get

$$
p_{0}=\frac{m C_{2}(\mu)}{4 \pi} \sqrt{8 \pi \kappa_{2}} \mathcal{A}_{2}\left[\kappa_{2}-\mu\right] .
$$

The equation for the bound-state momentum $\kappa_{2}$ takes the form

$$
1=\frac{m C_{0}(\mu)}{2 \pi}\left[\kappa_{2}-\mu\right]-\frac{m C_{2}(\mu)}{2 \pi} \kappa_{2}^{2}\left[\kappa_{2}-\mu\right] .
$$

Inserting eqs. (A.2), we can rewrite this expression as

$$
\kappa_{2}=\frac{1}{a}+\frac{r_{e}}{2} \kappa_{2}^{2}+O\left(\kappa_{2}^{4}\right) .
$$

The normalization condition,

$$
\int \frac{d^{d} \mathbf{q}}{(2 \pi)^{d}}\left(\frac{\sqrt{8 \pi \kappa_{2}} \mathcal{A}_{2}}{q^{2}+\kappa_{2}^{2}}+p_{0}\right)^{2}=1
$$

yields the following expression for the asymptotic normalization coefficient:

$$
\mathcal{A}_{2}^{-2}=1-\kappa_{2} r_{e} \frac{\left[\kappa_{2}-\mu\right]^{2}}{[1 / a-\mu]^{2}} .
$$

Using the equation $a \kappa_{2}=1+O\left(\kappa_{2}^{3}\right)$, one may rewrite the above equation as

$$
\mathcal{A}_{2}^{-2}=1-\kappa_{2} r_{e}+O\left(\kappa_{2}^{3}\right) \text {. }
$$

As we see, the final result for $\mathcal{A}_{2}$ does not depend on the regularization used.

\section{B Calculation of the integrals}

In order to calculate the integrals in eq. (3.15), it is convenient to change the integration variables

$$
\frac{\sqrt{3}}{2} x=\sinh u, \quad \frac{\sqrt{3}}{2} y=\sinh v .
$$

Then, $I_{0}=I_{1}+I_{2}$, where

$$
I_{1}=\frac{2}{\sqrt{3}} \int_{0}^{\infty} d u \frac{\sin ^{2}\left(s_{0} u\right)}{\cosh ^{2} u}=\frac{1}{\sqrt{3}}\left(1-\frac{\pi s_{0}}{\sinh \left(\pi s_{0}\right)}\right),
$$

where the last equality was obtained by using the equality given in ref. [51]

$$
\int_{0}^{\infty} \sin a x \frac{\sinh \beta x}{\cosh ^{2} \gamma x} d x=\frac{\pi\left(a \sin \frac{\beta \pi}{2 \gamma} \cosh \frac{a \pi}{2 \gamma}-\beta \cos \frac{\beta \pi}{2 \gamma} \sinh \frac{a \pi}{2 \gamma}\right)}{\gamma^{2}\left(\cosh \frac{a \pi}{\gamma}-\cos \frac{\beta \pi}{\gamma}\right)}
$$

with $a=s_{0}, \beta=i s_{0}, \gamma=1$. 
Using the same substitution, we obtain

$$
I_{2}=\frac{2}{\pi} \int_{-\infty}^{\infty} \int_{-\infty}^{\infty} d u d v \frac{\sin \left(s_{0} u\right) \sin \left(s_{0} v\right)}{\sinh ^{2} u+\sinh ^{2} v-\sinh u \sinh v+\frac{3}{4}} .
$$

It is convenient to define $w=u+v$ and $z=u-v$. Then,

$$
I_{2}=\frac{2}{\pi} \int_{-\infty}^{\infty} d z \cos \left(2 s_{0} z\right) J(z)
$$

where

$$
J(z)=\frac{4 \sqrt{3} \pi}{9}\left(\frac{1}{\cosh ^{2} z+3 \sinh ^{2} z}-\frac{2}{3 \cosh ^{2} z+\sinh ^{2} z}\right)
$$

The integral over the variable $z$ can again be performed, using the formula from ref. [51]

$$
\int_{0}^{\infty} \frac{\cos a x d x}{\cosh \beta x+\cos \gamma}=\frac{\pi}{\beta} \frac{\sinh \frac{a \gamma}{\beta}}{\sin \gamma \sinh \frac{a \pi}{\beta}}
$$

with $a=2 s_{0}, \beta=2$ and $\gamma=\frac{2 \pi}{3}$ or $\gamma=\frac{\pi}{3}$ (in the first and the second terms of eq. (B.6), respectively). At the end, one gets

$$
I_{2}=\frac{8 \pi}{9} \frac{1}{\sinh \left(\pi s_{0}\right)}\left(\sinh \frac{2 \pi s_{0}}{3}-2 \sinh \frac{\pi s_{0}}{3}\right)
$$

and the eq. (3.15) is reproduced.

\section{Wave function}

In this appendix we study the singularities of the wave function and show that, to the leading order, it can be pulled out from the integrals that determine the energy shift of the particle-dimer bound state. The wave function obeys eq. (3.8). The location of the singularities of $\Psi(p)$ in the complex- $p$ plane is determined, as usual, by the Landau equations. There are two types of singularities (note that the denominator $-a^{-1}+\sqrt{\frac{3 q^{2}}{4}+\kappa^{2}}$ does not vanish in the integration region):

\section{C.1 Endpoint singularities}

The argument of the logarithm is $a_{ \pm}(q)=p^{2} \pm p \cdot q+q^{2}+\kappa^{2}$. At $q=0$, the equation $a_{ \pm}(q=0)=0$ yields $p= \pm i \kappa$. Examine now this potential singularity in detail. Let us start, for instance, at $p=0$ and approach the singular point $p \rightarrow i \kappa$ along some path in the complex $p$-plane (for instance, along the path $p=i t+0.05 t(1-t), 0 \leq t \leq 1$ ). The four singularities of the logarithm, which are determined by the solutions of the equations $a_{ \pm}(q)=0$, travel along the lines

$$
q_{1,2}(p)=\frac{-p \pm i \sqrt{3 p^{2}+4 \kappa^{2}}}{2}, \quad q_{3,4}(p)=\frac{p \pm i \sqrt{3 p^{2}+4 \kappa^{2}}}{2}
$$



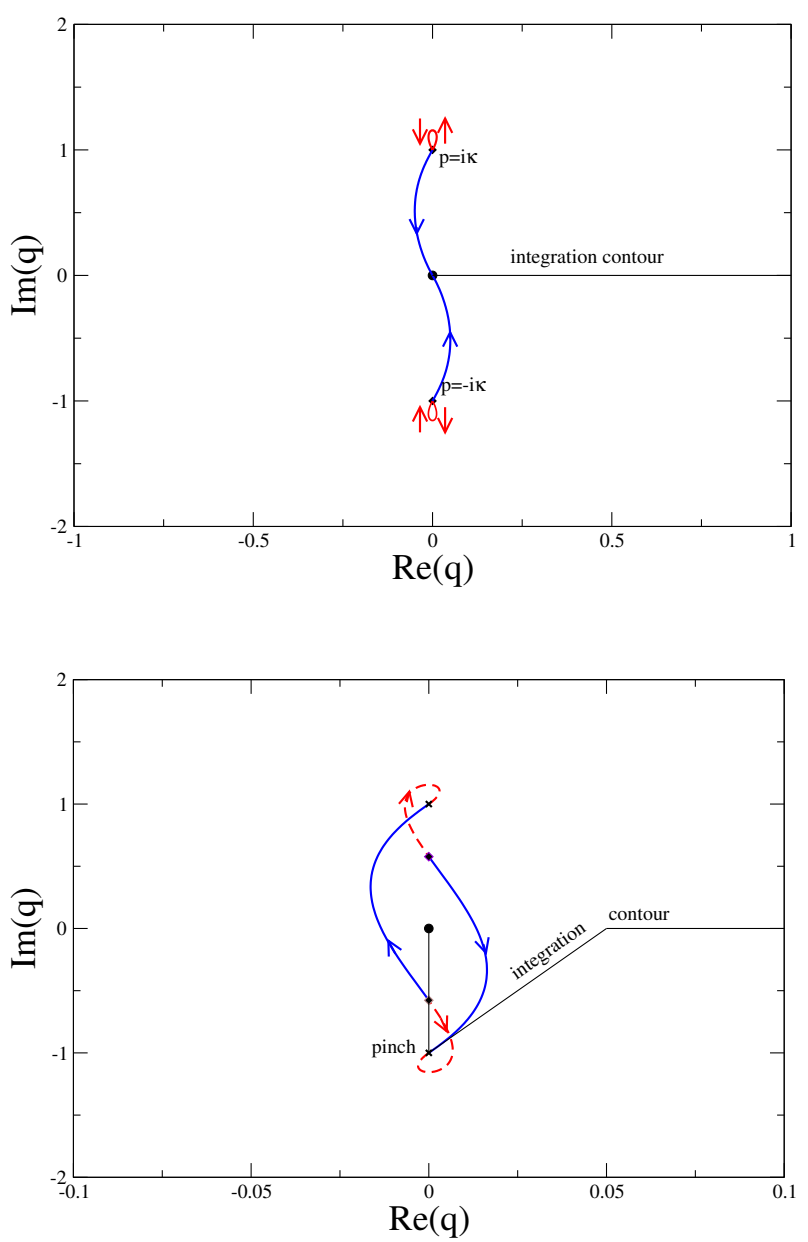

Figure 2. Trajectories of the singularities of the kernel of eq. (3.8) in the complex $q$-plane, Left panel: $p \rightarrow i \kappa$, right panel: $p \rightarrow \frac{2 i}{\sqrt{3}} \kappa$.

whereas the singularity of the denominator given by the equation $-a^{-1}+\sqrt{\frac{3}{4} q^{2}+\kappa^{2}}=0$ stays fixed in the in the complex $q$-plane. The trajectories $q_{1,2,3,4}$ are shown schematically in figure 2, left panel. Two singularities travel from $q= \pm i \kappa$ towards $q=0$ and two others return to $q= \pm i \kappa$. The contour deformation is not needed. Substituting now $p= \pm i \kappa$ into the kernel, we get

$$
\pm \frac{1}{2 i \kappa q} \ln \frac{ \pm i \kappa+q}{\mp i \kappa+q}=\frac{1}{\kappa q} \arctan \frac{\kappa}{q}
$$

The logarithm is indeed singular at $q=0$, but the integral over $q$ exists, due to the presence of an additional factor $q^{2}$. Consequently, there is no singularity at $p= \pm i \kappa$.

Further, at $q=\Lambda$, we get the equation $p^{2} \pm p \Lambda+\Lambda^{2}+\kappa^{2}=0$. The solution of this equation gives $p= \pm \frac{1}{2}\left(\Lambda \pm \sqrt{\Lambda^{2}-4\left(\Lambda^{2}+\kappa^{2}\right)}\right)$. These points are located very far from the origin and should not be taken into account. 


\section{C.2 Pinch singularities}

In order to find the location of the pinch singularities, we have to solve the equation

$$
\frac{d}{d q}\left(p^{2} \pm p q+q^{2}+\kappa^{2}\right)=0
$$

which gives $p= \pm 2 q$. Substituting this back to the argument of the logarithm, we get $a_{ \pm}(q)=\left(q \pm \frac{p}{2}\right)^{2}+\frac{3}{4} p^{2}+\kappa^{2}$, i.e., the argument vanishes at $p= \pm \frac{2 i}{\sqrt{3}} \kappa$.

Consider again the trajectories of the singularities of the logarithm in the complex $q$-plane, when $p$ varies, according to, for instance, along the path $p=2 / \sqrt{3}(i t+0.05 t(1-$ $t)$ ), $0 \leq t \leq 1$. These trajectories are shown in figure 2 , right panel. In this case, it is necessary to deform the integration contour, in order to avoid the singularities of the logarithm. At $p= \pm \frac{2 i}{\sqrt{3}} \kappa$ the contour gets pinched between two singularities. It is, however, straightforward to check that the singularity of the integrand along the new integration contour is of an integrable type: logarithmic for $a^{-1} \neq 0$ and of square-root type for $a^{-1}=0$. Consequently, the function $\Psi(p)$, defined by this integral, is non-singular there (albeit the derivatives become, in general, singular).

Open Access. This article is distributed under the terms of the Creative Commons Attribution License (CC-BY 4.0), which permits any use, distribution and reproduction in any medium, provided the original author(s) and source are credited.

\section{References}

[1] N. Mathur et al., Roper resonance and S(11)(1535) from lattice QCD, Phys. Lett. B 605 (2005) 137 [hep-ph/0306199] [INSPIRE].

[2] D. Guadagnoli, M. Papinutto and S. Simula, Extracting excited states from lattice QCD: the Roper resonance, Phys. Lett. B 604 (2004) 74 [hep-lat/0409011] [INSPIRE].

[3] D.B. Leinweber et al., Baryon spectroscopy in lattice QCD, Lect. Notes Phys. 663 (2005) 71 [nucl-th/0406032] [INSPIRE].

[4] K. Sasaki, S. Sasaki and T. Hatsuda, Spectral analysis of excited nucleons in lattice QCD with maximum entropy method, Phys. Lett. B 623 (2005) 208 [hep-lat/0504020] [INSPIRE].

[5] K. Sasaki and S. Sasaki, Excited baryon spectroscopy from lattice QCD: Finite size effect and hyperfine mass splitting, Phys. Rev. D 72 (2005) 034502 [hep-lat/0503026] [INSPIRE].

[6] T. Burch et al., Excited hadrons on the lattice: baryons, Phys. Rev. D 74 (2006) 014504 [hep-lat/0604019] [INSPIRE].

[7] K.-F. Liu, Y. Chen, M. Gong, R. Sufian, M. Sun and A. Li, The Roper puzzle, PoS (LATTICE 2013) 507 [arXiv: 1403.6847] [INSPIRE].

[8] M.S. Mahbub, A.O. Cais, W. Kamleh, D.B. Leinweber and A.G. Williams, Positive-parity excited-states of the nucleon in quenched lattice QCD, Phys. Rev. D 82 (2010) 094504 [arXiv: 1004.5455] [INSPIRE].

[9] B.G. Lasscock et al., Even parity excitations of the nucleon in lattice QCD, Phys. Rev. D 76 (2007) 054510 [arXiv:0705.0861] [INSPIRE]. 
[10] C.B. Lang, L. Leskovec, M. Padmanath and S. Prelovsek, Pion-nucleon scattering in the Roper channel from lattice QCD, Phys. Rev. D 95 (2017) 014510 [arXiv:1610.01422] [INSPIRE].

[11] S.R. Beane, W. Detmold, K. Orginos and M.J. Savage, Nuclear physics from lattice QCD, Prog. Part. Nucl. Phys. 66 (2011) 1 [arXiv:1004.2935] [InSPIRE].

[12] NPLQCD collaboration, S.R. Beane et al., Light nuclei and hypernuclei from quantum chromodynamics in the limit of SU(3) flavor symmetry, Phys. Rev. D 87 (2013) 034506 [arXiv:1206.5219] [INSPIRE].

[13] NPLQCD collaboration, E. Chang et al., Magnetic structure of light nuclei from lattice QCD, Phys. Rev. D 92 (2015) 114502 [arXiv:1506.05518] [InSPIRE].

[14] E. Epelbaum, H. Krebs, D. Lee and U.-G. Meissner, Lattice effective field theory calculations for $A=3,4,6,12$ nuclei, Phys. Rev. Lett. 104 (2010) 142501 [arXiv:0912.4195] [INSPIRE].

[15] E. Epelbaum, H. Krebs, D. Lee and U.-G. Meissner, Ab initio calculation of the Hoyle state, Phys. Rev. Lett. 106 (2011) 192501 [arXiv:1101.2547] [INSPIRE].

[16] A. Rokash, E. Epelbaum, H. Krebs, D. Lee and U.-G. Meißner, Finite volume effects in low-energy neutron-deuteron scattering, J. Phys. G 41 (2014) 015105 [arXiv:1308.3386] [INSPIRE].

[17] S. Elhatisari et al., Ab initio $\alpha$ - $\alpha$ scattering, Nature 528 (2015) 111 [arXiv:1506.03513] [INSPIRE].

[18] K. Polejaeva and A. Rusetsky, Three particles in a finite volume, Eur. Phys. J. A 48 (2012) 67 [arXiv: 1203.1241] [INSPIRE].

[19] U.-G. Meißner, G. Ríos and A. Rusetsky, Spectrum of three-body bound states in a finite volume, Phys. Rev. Lett. 114 (2015) 091602 [arXiv: 1412.4969] [INSPIRE].

[20] P. Guo, One spatial dimensional finite volume three-body interaction for a short-range potential, Phys. Rev. D 95 (2017) 054508 [arXiv:1607.03184] [INSPIRE].

[21] P. Guo and V. Gasparian, An solvable three-body model in finite volume, arXiv: 1701.00438 [INSPIRE].

[22] R.A. Briceno and Z. Davoudi, Three-particle scattering amplitudes from a finite volume formalism, Phys. Rev. D 87 (2013) 094507 [arXiv: 1212.3398] [INSPIRE].

[23] M.T. Hansen and S.R. Sharpe, Relativistic, model-independent, three-particle quantization condition, Phys. Rev. D 90 (2014) 116003 [arXiv:1408.5933] [InSPIRE].

[24] M.T. Hansen and S.R. Sharpe, Expressing the three-particle finite-volume spectrum in terms of the three-to-three scattering amplitude, Phys. Rev. D 92 (2015) 114509 [arXiv: 1504.04248] [INSPIRE].

[25] M.T. Hansen and S.R. Sharpe, Perturbative results for two and three particle threshold energies in finite volume, Phys. Rev. D 93 (2016) 014506 [arXiv:1509.07929] [INSPIRE].

[26] M.T. Hansen and S.R. Sharpe, Threshold expansion of the three-particle quantization condition, Phys. Rev. D 93 (2016) 096006 [arXiv: 1602.00324] [INSPIRE].

[27] M.T. Hansen and S.R. Sharpe, Applying the relativistic quantization condition to a three-particle bound state in a periodic box, Phys. Rev. D 95 (2017) 034501 [arXiv: 1609.04317] [INSPIRE]. 
[28] R.A. Briceño, M.T. Hansen and S.R. Sharpe, Relating the finite-volume spectrum and the two-and-three-particle $S$ matrix for relativistic systems of identical scalar particles, Phys. Rev. D 95 (2017) 074510 [arXiv: 1701.07465] [INSPIRE].

[29] M. Lüscher, Two particle states on a torus and their relation to the scattering matrix, Nucl. Phys. B 354 (1991) 531 [INSPIRE].

[30] S. Kreuzer and H.W. Hammer, The triton in a finite volume, Phys. Lett. B 694 (2011) 424 [arXiv: 1008.4499] [INSPIRE].

[31] S. Kreuzer and H.W. Hammer, On the modification of the Efimov spectrum in a finite cubic box, Eur. Phys. J. A 43 (2010) 229 [arXiv:0910.2191] [inSPIRE].

[32] S. Kreuzer and H.W. Hammer, Efimov physics in a finite volume, Phys. Lett. B 673 (2009) 260 [arXiv:0811.0159] [INSPIRE].

[33] S. Kreuzer and H.W. Grießhammer, Three particles in a finite volume: the breakdown of spherical symmetry, Eur. Phys. J. A 48 (2012) 93 [arXiv:1205.0277] [INSPIRE].

[34] P.F. Bedaque, H.W. Hammer and U. van Kolck, Renormalization of the three-body system with short range interactions, Phys. Rev. Lett. 82 (1999) 463 [nucl-th/9809025] [INSPIRE].

[35] P.F. Bedaque, H.W. Hammer and U. van Kolck, The three boson system with short range interactions, Nucl. Phys. A 646 (1999) 444 [nucl-th/9811046] [INSPIRE].

[36] E. Epelbaum, J. Gegelia, U.-G. Meißner and D.-L. Yao, Renormalization of the three-boson system with short-range interactions revisited, Eur. Phys. J. A 53 (2017) 98 [arXiv: 1611.06040] [INSPIRE].

[37] H.W. Hammer, J.Y. Pang and A. Rusetsky, Three particle quantization condition in a finite volume: 2. general formalism and the analysis of data, arXiv:1707.02176 [INSPIRE].

[38] M. Lüscher, Volume dependence of the energy spectrum in massive quantum field theories. 1. Stable particle states, Commun. Math. Phys. 104 (1986) 177 [INSPIRE].

[39] Z. Davoudi and M.J. Savage, Improving the volume dependence of two-body binding energies calculated with lattice QCD, Phys. Rev. D 84 (2011) 114502 [arXiv:1108.5371] [InSPIRE].

[40] S. Weinberg, Evidence that the deuteron is not an elementary particle, Phys. Rev. 137 (1965) B672 [INSPIRE].

[41] D.B. Kaplan, More effective field theory for nonrelativistic scattering, Nucl. Phys. B 494 (1997) 471 [nucl-th/9610052] [INSPIRE].

[42] C. Itzykson and J.B. Zuber, Quantum field theory, McGraw-Hill, U.S.A. (1980).

[43] G.V. Skornyakov and K.A. Ter-Martirosyan, Three body problem for short range forces. I. Scattering of low energy neutrons by deuterons, Zh. Eksp. Teor. Fiz. 31 (1956) 775 [Sov. Phys. JETP 4 (1956) 648].

[44] G.S. Danilov, On the three-body problem with short-range forces, Zh. Eksp. Teor. Fiz. 40 (1961) 498 [Sov. Phys. JETP 13 (1961) 349].

[45] R.A. Minlos and L.D. Faddeev, Comment on the problem of three particles with point interactions, Zh. Eksp. Teor. Fiz. 41 (1961) 1850 [Sov. Phys. JETP 14 (1961) 1315].

[46] V. Efimov, Energy levels arising form the resonant two-body forces in a three-body system, Phys. Lett. B 33 (1970) 563. 
[47] V.N. Efimov, Weakly-bound states of 3 resonantly-interacting particles, Sov. J. Nucl. Phys. 12 (1971) 589 [Yad. Fiz. 12 (1970) 1080]. [INSPIRE].

[48] V. Efimov, Energy levels of three resonantly interacting particles, Nucl. Phys. A 210 (1973) 157 [INSPIRE].

[49] S. König and D. Lee, Volume dependence of N-body bound states, arXiv:1701.00279 [INSPIRE].

[50] D.B. Kaplan, M.J. Savage and M.B. Wise, A new expansion for nucleon-nucleon interactions, Phys. Lett. B 424 (1998) 390 [nucl-th/9801034] [INSPIRE].

[51] I.S. Gradsteyn and I.M. Ryzhik, Table of Integrals, Series, and Products, $8^{\text {th }}$ edition, Academic Press, New York U.S.A. (2014). 